\title{
VÍA DEL NACIMIENTO EN GEMELAR MONOCORIÓNICO CON TRANSFUSIÓN INTERG EMELAR Y TERAPIA IN UTERO
}

\author{
Jorge Hasbun H. ${ }^{1}$, Waldo Sepúlveda L. ${ }^{2}$, Mauro Parra C. ${ }^{1}$ \\ ${ }^{1}$ Departamento de Obstetricia y Ginecología, Unidad de Medicina Fetal, Hospital Clínico J.J. Aguirre, Universidad de \\ Chile. ${ }^{2}$ Departamento de Obstetricia y Ginecología, Unidad de Cirugía Fetal, Clínica Las Condes.
}

\section{RESUMEN}

El propósito de la comunicación es revisar la vía del parto en embarazo gemelar complicado por Síndrome de Transfusión Gemelo-Gemelo (STGG) y por gemelo acardio con perfusión arterial reversa. La placentación del gemelar monocoriónico por sus anastomosis arteriales y venosas genera esas complicaciones que se asocian a una mortalidad cercana a 100\% cuando no son tratadas en el período antenatal. Se describen dos casos de gemelar monocoriónico complicado, uno con STGG y otro, un acardio con perfusión reversa, ambos con diagnóstico ultrasónico precoz en primer trimestre y comienzo del segundo, que fueron exitosamente tratados in útero con coagulación láser sobre anastomosis vascular placentaria y sobre arteria umbilical pelviana obteniendo mejoría hemodinámica y recuperación de la funcionalidad placentaria. Se discuten aspectos generales del cuidado médico antenatal e intraparto del embarazo gemelar y específicamente del gemelar monocoriónico complicado. Se concluye que la terapia in útero fue exitosa además de mejorar el pronóstico puede favorecer la vía vaginal de parto.

\section{PALABRAS CLAVE: embarazo gemelar monocoriónico, anastomosis vasculares, terapia láser, parto vaginal}

\section{SUMMARY}

The aim of this communication is to review the way of delivering twin pregnancy complicated by either Twin to Twin Transfusion Syndrome (TTTS) or acardic twin with reverse arterial perfusion. The monochorionic placentae is characterised by superficial or deep vascular anastomosis which leads to the complications mentioned above, which are associated with almost $100 \%$ mortality when they are not treated antenatally. We describe two cases of severe TTTS and acardic twin with reverse arterial perfusion both diagnosed antenatally during the first and early second trimester of pregnancy on which laser treatment either on placental vascular anastomoses or pelvic single umbilical artery were done successfully. In addition of this we discuss some clinical aspects of prevention of premature delivery, antenatal and intrapartum medical care for twin pregnancy in general and specifically for complicated monochorionic twin pregnancy. We conclude that a successful fetal therapy improves pregnancy prognosis and makes possible vaginal delivery.

KEY WORDS: Monochorionic twin pregnancy, vascular anastomoses, laser therapy, vaginal delivery 


\section{INTRODUCCIÓN}

El embarazo gemelar genera mortalidad perinatal en $12-14 \%$ y prematurez extrema en el $16 \%$ (1). Las complicaciones determinantes ocurren en el período antenatal, intraparto o postparto. Los riesgos intraparto incluyen parto distócico, complicaciones funiculares, asfixia fetal, gemelos en colisión y cesárea de urgencia. Por magnitud del riesgo y el contexto médico-legal hay tasa alta de cesáreas: $75 \%$ en la Maternidad del Hospital Clínico en el período 1992-2000 (2). El 25\% asistido por parto vaginal está limitado a gemelos dicoriónicos y algunos casos con criterios tradicionales.

El nacimiento gemelar tiene un gran potencial de complicaciones y su manejo se optimiza en centros terciarios con recursos y personal capacitado en protocolos que incluyen el conocimiento de la corionicidad y las anomalías de la placentación (3).

El gemelar monocoriónico tiene morbimortalidad perinatal tres veces mayor que en dicoriónicos, especialmente relacionada a comunicaciones vasculares intraplacentarias que generan transfusión intergemelar a través de anastomosis arteriovenosa (Aa-v), con retorno por anastomosis arterioarterial $(\mathrm{Aa}-\mathrm{a})$ en respuesta a diferencias de presión arterial $(4,5,6,7)$. La presencia de Aa-v, no compensada por Aa-a protectora (7), ante diferencia de presión intraplacentaria, origina transfusión unidireccional, de mal pronóstico perinatal y su expresión máxima es el Síndrome de Transfusión Gemelo-Gemelo (STGG) grave, presente en 15\% de los casos y que tiene evolución letal $(8,9)$.

El diagnóstico de monocorionicidad y el ultrasonido (US) han facilitado el acceso a la unidad fetoplacentaria con fines terapéuticos. Actualmente, hay estudios prospectivos de tratamiento antenatal de transfusión intergemelar con cirugía láser que permiten la coagulación de las anastomosis en placenta o en cordón umbilical, revirtiendo los cambios hemodinámicos secundarios (10) con mejoría del pronóstico perinatal $(11,12,13)$.

Aunque la corrección in útero de la anomalía y la mejoría hemodinámica fetal renuevan posibilidades de manejo intraparto, no hay referencias sobre la vía y se asume que generalmente se indica cesárea. En nuestro medio se han comunicado seis pacientes de monocoriónico con transfusión y terapia in útero, los cuáles tuvieron parto por cesárea $(14,15,16,17,18)$.

El propósito de esta comunicación es describir dos casos clínicos de embarazo gemelar monocoriónicos con transfusión gemelo-gemelo, sometidos a terapia antenatal no complicada, y que su manejo prospectivo permitió indicar vía vaginal.

\section{CASOS CLÍNICOS}

Caso 1. Paciente de 28 años, G2P0A1, con antecedentes de Púrpura Trombocitopénico Idiomático y Enfermedad de Von Willebrand, esplenectomizada. En semana 11 se confirma embarazo gemelar monocorial biamniótico y se inicia protocolo de pesquisa STGG. En semana 16 hay secuencia oligo-polihidroamnios, discordancia del tamaño vesical y del tamaño fetal con Gem A de 210 gramos (receptor) y Gem B de 179 gramos (dador), con doppler umbilical del dador y ductus venoso del receptor normales (Figuras 1 y 2).

En semana 17 y 18 se confirmó STGG con indicación de coagulación endoscópica. Se coordinó traslado a Centro especializado (Harris Birthright Research Centre for Fetal Medicine, King's College Hospital de Londres). En ese Centro, en semana 20 , se efectuó coagulación intraplacentaria de

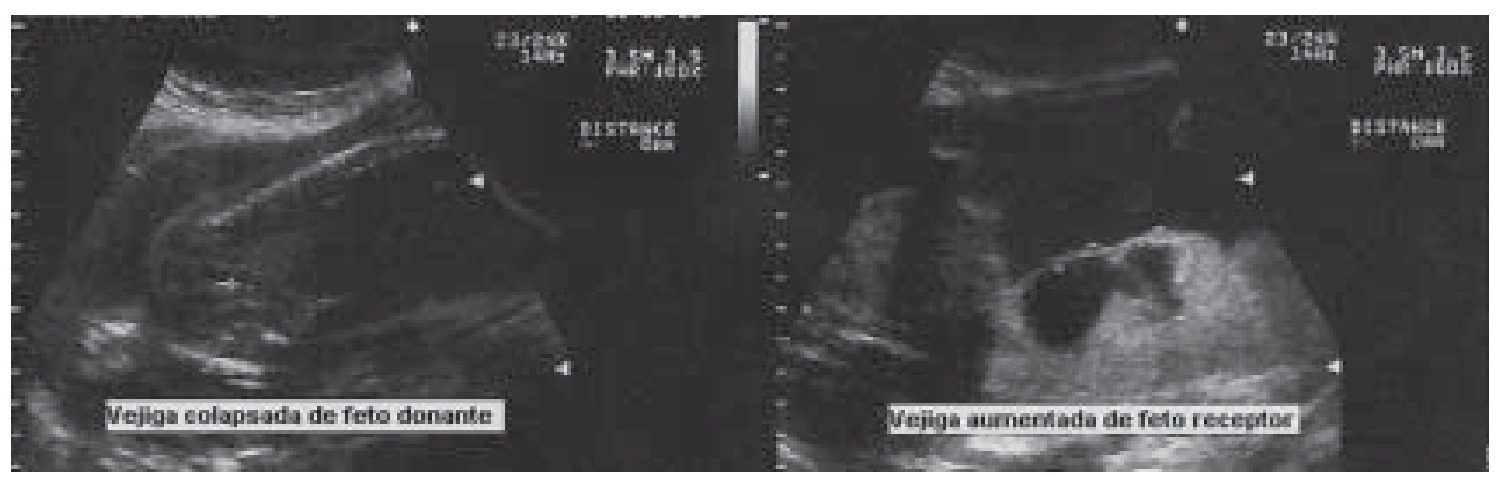

Figura 1. Síndrome de transfusión gemelo a gemelo (STGG): Vejiga pequeña en gemelo dador y vejiga aumentada en gemelo receptor en semana 15. Caso 1. 


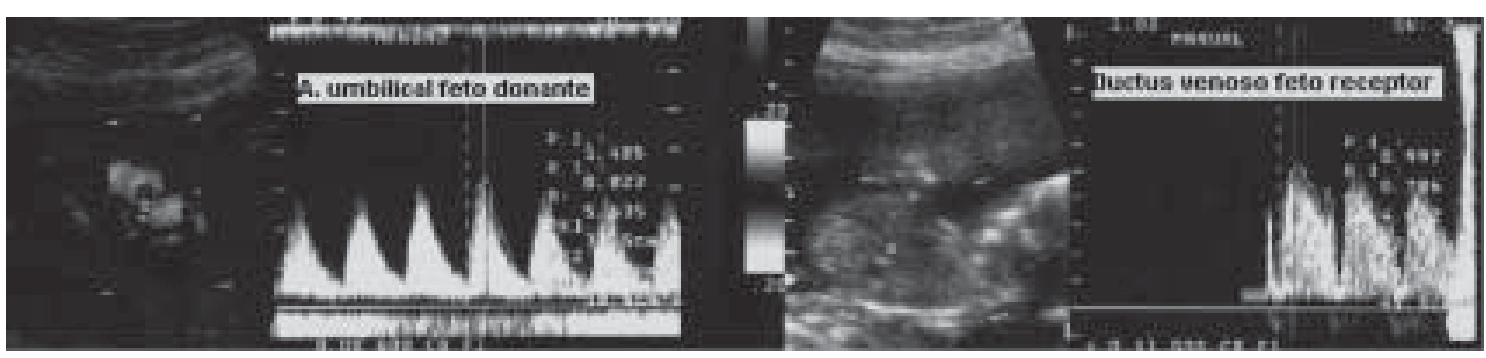

Figura 2. Doppler de arteria umbilical y ductus venoso normales del feto donante y del receptor, semana 15. Caso 1.

Tabla I

\section{CRECIMIENTO FETAL DE GEM A - GEM B EN SÍNDROME DE TRANSFUSIÓN GEMELO A GEMELO.} CASO 1

\begin{tabular}{lrrrrrrrrrrrr}
\hline Semana & \multicolumn{2}{c}{16} & \multicolumn{2}{c}{20} & \multicolumn{2}{c}{23} & \multicolumn{2}{c}{26} & \multicolumn{2}{c}{28} & & \\
& $G A$ & $G B$ & $G A$ & $G B$ & $G A$ & $G B$ & $G A$ & $G B$ & $G A$ & $G B$ & $G A$ & $G B$ \\
\hline DBP & 38 & 36 & 48 & 44 & 63 & 65 & 69 & 67 & 74 & 80 & 89 & 89 \\
CC & 138 & 130 & 163 & 162 & 234 & 237 & 253 & 250 & 279 & 292 & 322 & 316 \\
CA & 126 & 108 & 153 & 132 & 215 & 209 & 231 & 231 & 261 & 258 & 289 & 308 \\
Fémur & 21 & 21 & 29 & 28 & 44 & 45 & 50 & 49 & 57 & 55 & 65 & 64 \\
Peso & 210 & 179 & 350 & 275 & 823 & 811 & 1065 & 1033 & 1502 & 1482 & 2346 & 2221 \\
Talla & 20,2 & 20,2 & 32,9 & 36,2 & 36,2 & 36.2 & 40,0 & 40.0 & 44,4 & 44.4 & & \\
\hline
\end{tabular}

anastomosis (Prof. K. Nicolaides), sin complicaciones. El control demostró fetos vivos, vejigas llenas y Doppler umbilical normal. Retorna al 6ํㅡa y en controles seriados hay hemodinamia estable, sin reaparición de signos de transfusión y crecimiento concordante (Tabla I), (Figuras 3 y 4). Se mantiene en reposo en su domicilio con autocontrol de actividad uterina.

Se hospitaliza por inicia dinámica uterina en semana 34 , se indica profilaxis de membrana

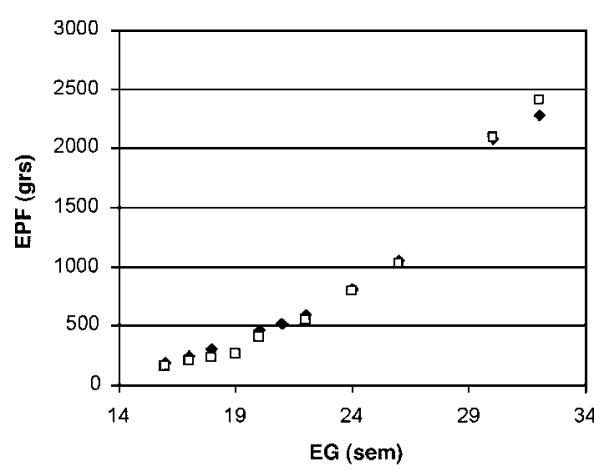

Figura 3. Curva de peso fetal estimado. Gem A y B. Caso 1. hialina y ácido tranexámico. Con ambos fetos en cefálica se permite vía vaginal.

Conducción con anestesia epidural en $7 \mathrm{~cm}$, ocitocina y RAM. El período de dilatación duró 10 horas con Gem A en OIDP en $\mathrm{S}+1$. Se aplicó rama derecha de Forceps Kjelland, rotando presentación a OP con desprendimiento cefálico expedito, se obtuvo RN masculino de $2580 \mathrm{~g}$, Apgar 9-9. Gemelo B, en situación alta, con bolsa de aguas prominente, requirió de rotura artificial de membranas controlada; el descenso y el parto fueron inmediatos, y no requirió de maniobras. Se

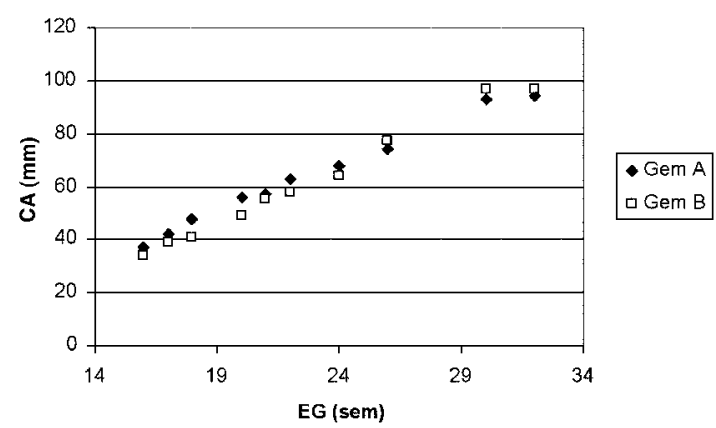

Figura 4. Curva de crecimiento de la circunferencia abdominal (CA): Gem A y B. Caso 1. 


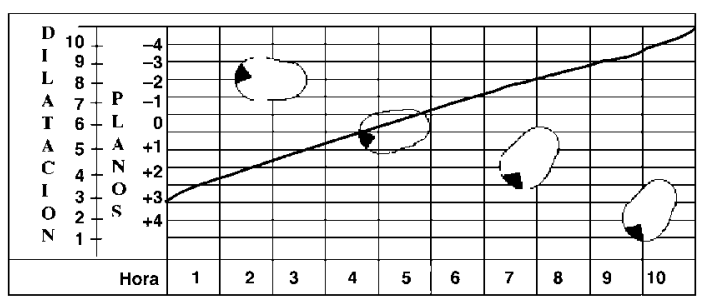

Figura 5. Curvas de dilatación y progresión del descenso en OIDP. Gem A. Caso 1.

obtuvo un RN masculino de $2520 \mathrm{~g}$, Apgar 9-9. Placenta monocorial biamniótica de $980 \mathrm{~g}$. Gemelos y madre evolucionan satisfactoriamente. El partograma se muestra en la Figura 5.

Caso 2. Paciente de 25 años, G1P0. Se confirma en semana 12 embarazo gemelar monocorial biamniótico, con gemelo de $66 \mathrm{~mm}$ de longitud céfalo-nalgas y 175 latidos cardíacos/minuto; en segundo saco se aprecia masa amorfa de $44 \mathrm{~mm}$ de longitud, sin actividad cardiaca y sin definición de polo cefálico. Doppler demostró flujo arterial umbilical hacia la masa orientando el diagnóstico a feto acardio con perfusión reversa, excluyendo muerte embrionaria de co-gemelo. En controles posteriores se demostró gemelo bomba con crecimiento normal y acardio con escaso desarrollo, con quiste dorsal y movimientos de extremidades inferiores. Se planteó posibilidad de constricción espontánea de anastomosis y manejo expectante. En semana 25 inicia dolor abdominal, taquipnea y aumento brusco de altura, tono y contracciones uterinas. El ultrasonido revela crecimiento corporal del acardio $(20 \mathrm{~cm})$, oligoamnios y cordones cercanos. El gemelo bomba, con crecimiento adecuado, apremio hemodinámico (Figura 6), vejiga distendida, cordón edematoso y polihidroamnios. Se identificó la Aa-a entrando a nivel de la inserción placentaria del cordón del acardio. Uno de los autores (WS) efectuó ablación láser intrafetal, logrando oclusión expedita del flujo. En control al 5o día se observó regresión del acardio $(16 \mathrm{~cm})$, pero con polihidroamnios persistente y protrusión de membranas por el orificio cervical interno, acortamiento cervical a $7 \mathrm{~mm}$ y útero irritable (Figura 7). Se complementa terapia con amniodrenaje, extrayéndose 2200 cc de líquido amniótico. Se mantuvo la paciente con reposo en domicilio y autocontrol de contractilidad hasta la semana 36 . Ingresa en trabajo de parto con dilatación cervical de $3 \mathrm{~cm}$, feto reactivo en presentación cefálica alta y oligoamnios. Se decide conducción ocitócica de vía vaginal. A los $8 \mathrm{~cm}$ de dilatación presentó

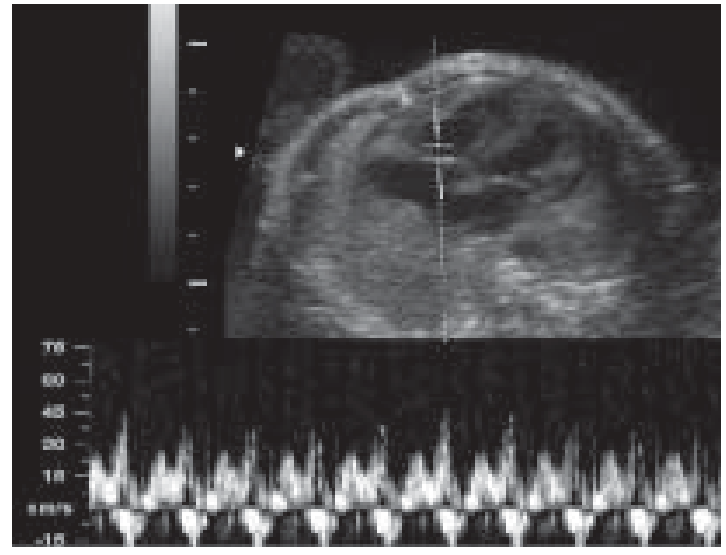

Figura 6. Gem dador con cardiomegalia, engrosamiento de tabique y paredes ventriculares. Regurgitación tricuspídea, visible en Doppler por onda reversa. Caso 2.

rotura espontánea de membranas, con salida escaso de líquido amniótico claro. Dilatación completa en 9 horas, feto en presentación cefálica, OIDP deflectada, en espinas 0 . Fué intervenida con fórceps Kjelland aplicando solo rama derecha, efectuando rotación a occipitopúbica con descenso y desprendimiento sin dificultad. Se obtuvo RN femenino de $2820 \mathrm{~g}$, Apgar 9-9, acardio de $324 \mathrm{~g}$. Placenta monocorial biamniótica de $715 \mathrm{~g}$. El partograma se muestra en la Figura 8.

\section{DISCUSIÓN}

Por variabilidad natural de anastomosis y repartición placentaria en monocoriónico las posibilidades de evolución clínica son:

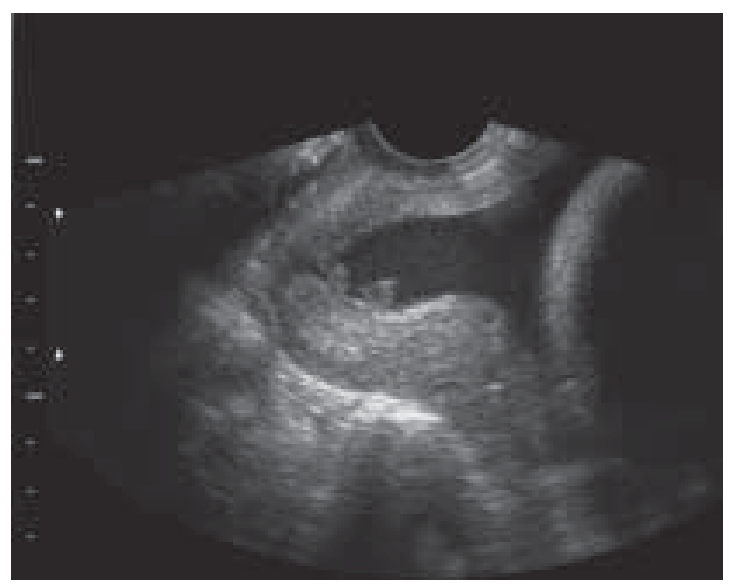

Figura 7. Gem dador con hidroamnios, protrusión de membranas en $\mathrm{OCl}$ y acortamiento cervical a $7 \mathrm{~mm}$. Caso 2. 


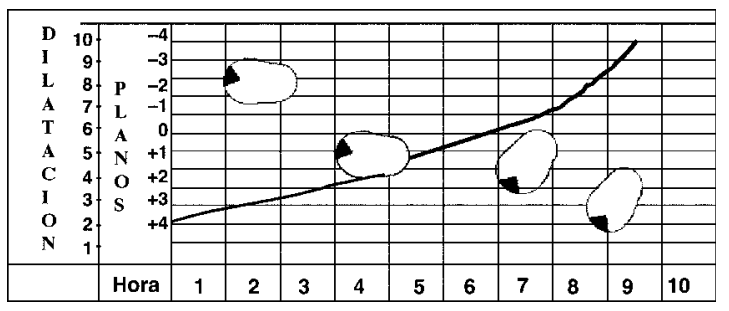

Figura 8. Curvas de dilatación y progresión del descenso en OIDP. Gem A. Caso 2.

a) transfusión gemelo-gemelo crónica y grave si hay Aa-v no compensada,

b) transfusión aguda periparto en que un volumen importante es transfundido por una $\mathrm{Aa}-\mathrm{a}$ o $A v-v$, a la muerte de un gemelo o después del clampeo del cordón al parto del primero,

c) gran Aa-a o v-v o a-v con perfusión reversa desde un sano a un acardio,

d) restricción selectiva de crecimiento por repartición desigual de placenta, sin transfusión cuando no hay anastomosis,

e) placenta con igual repartición y ausencia de anastomosis, y

f) placenta bilobar que puede funcionar como dicoriónica.

Los dos casos presentados corresponden a las alternativas a y $\mathrm{c}$ de placentación y el buen resultado obtenido puede interpretarse por tres factores del cuidado médico: diagnóstico ultrasónico precoz, terapia antenatal eficaz y prevención de prematurez que lograron funcionalidad placentaria y madurez suficientes para acceder a parto vaginal.

1. Diagnóstico y terapia antenatal. La mortalidad es $80 \%$ en STGG y $50 \%$ en bomba de acardico (19) y el tratamiento utilizado en la segregación circulatoria fue el más actual: coagulación láser intraplacentaria endoscópica (caso 1) y ablación láser intrafetal con aguja guiada por ultrasonido (caso 2). Estos procedimientos han bajado el riesgo perinatal a la mitad, pero que están en evolución ya que aún es común el daño residual en sobrevivientes. El pronóstico mejora con su uso precoz, antes de complicaciones irreversibles.

El diagnóstico y el tratamiento en caso 1 fueron oportunos (semanas 16 y 20). El objetivo de la coagulación de la anastomosis culpable es revertir los cambios hemodinámicos disminuyendo el sobreaporte al corazón derecho del receptor y aumentando el volumen venoso en el dador (10). Se obtuvo la recuperación funcional de la placenta demostrada por crecimiento gemelar concordante y bienestar fetal hasta el parto en semana 34 . Se destaca la observación en las biometrías que nunca hubo más de $15 \%$ de diferencia en el peso, hecho importante porque que no tiene relación con el diagnóstico o pronóstico en el síndrome. El diagnóstico inicial, la coordinación con el Centro y el seguimiento ultrasónico posterior la efectuó uno de los autores (MP).

En el acardio, con diagnóstico precoz en semana 12, planteamos un manejo expectante por su escaso tamaño en tres controles, lo que sugería la constricción del vaso anastomótico. Sullivan y cols (20) recientemente presentan una serie con sobrevivencia de $9 / 10$ casos y cese espontáneo del flujo e involución (4 acardios menores de $125 \mathrm{~g}$ con sus bombas entre 2445 a $3825 \mathrm{~g}$ ), cuestionando el mal pronóstico previamente comunicado. Sin embargo, en semana 25 se observó un gran crecimiento del acardio, hidroamnios y compromiso hemodinámico del gemelo bomba; así la ventana de observación entre el diagnóstico inicial (semana 12) y el desarrollo del polihidroamnios (semana 25) fue de riesgo, y el lapso de observación refleja la carencia de predicción para decidir la ablación.

Se han propuesto para el manejo de estos casos la razón peso acardio/peso bomba (21) y la evaluación de la resistencia al flujo: gran diferencia en el índice tendría mejor pronóstico (menor flujo al acardio) (22), pero se mantiene la controversia entre tratamiento preventivo (semana 16) o tratamiento curativo a la aparición de complicaciones (insuficiencia cardiaca del feto dador).

La ablación intrafetal, original de Sepúlveda (23), tiene acceso más ventajoso a los vasos abdominales del acardio y mejores resultados que la oclusión endoscópica en asa libre de cordón (24, 25); el procedimiento fue expedito confirmándose el cese completo del flujo anastomótico, la resolución del apremio hemodinámico y el crecimiento fetal normal (percentil 40) hasta la semana 36 del nacimiento.

De los procedimientos en ambos casos, se destaca la ausencia de rotura iatrogénica de membranas antes de la semana 32; eso fue esencial en la evolución sin prematurez y en el acceso al nacimiento vaginal, ya que la complicación ocurre en $23 \%$ de las ablaciones y en $60 \%$ de las endoscopias $(24,26)$.

La evaluación del tratamiento es prospectiva porque incluye la normalidad neurológica en el desarrollo neonatal; una secuela neurológica puede significar indicación tardía o coagulación insuficiente. Hay que considerar que el daño gemelos/ únicos es 7/1 (27) y no es claro el efecto de la 
coagulación (13\% parálisis cerebral en sobrevivientes) (28).

El tratamiento logró recuperación funcional placentaria (crecimiento, madurez, tolerancia al parto) y la evaluación neurológica era normal hasta esta el momento de la comunicación; además siendo la génesis del daño probablemente por combinación de factores (restricción del crecimiento fetal, prematurez, muy bajo peso), la terapia efectuada podría ser considerada exitosa por la prevención de estos factores.

2. Prevención de prematurez. Más precoz y frecuente que en únicos (29), la prematurez amenaza en el parto al $50 \%$ de los gemelos (30) y es causa primaria de mortalidad (31), por lo que se planificó la prevención desde el segundo trimestre aplicando dos principios: a) educación integral en prematurez a las pacientes, incluyendo intervención en su estilo de vida, b) erradicación de factores asociados y c) manejo individual con percepción materna de contracciones, porque la aplicación sistemática de monitoreo domiciliario es difícil de implementar y con la hospitalización preventiva hay evidencias contradictorias.

El reconocimiento por la paciente del aumento de la contractilidad propia del gemelar (32) y el aumento en los 3 días previos al parto prematuro $(33,34)$ es útil en el manejo individual para decidir la hospitalización $(35,36)$ y tiene buenos resultados prospectivos $(37,38,39)$. Se utilizó el autocontrol materno dos veces por día que demostró contractilidad bajo 4/hora durante ocho semanas en caso 1 y diez semanas en caso 2; su incremento coincidió exactamente con el inicio del trabajo de parto. La pauta fue eficaz porque evitó hospitalizaciones por sobrediagnóstico (se internaron solo para cirugía antenatal) y fue predictivo en la consulta que determinó hospitalización definitiva por parto.

En el caso 2, luego de la ablación, hubo mejoría hemodinámica rápida del gemelo bomba, pero en el control postoperatorio se detectaron contracciones e hidroamnios persistente. La interpretación fue que la reabsorción del líquido amniótico en las membranas y la deglución-diuresis son mecanismos adaptativos del gemelo bomba, más tardíos que su respuesta circulatoria a la ablación y la conclusión fue que un amniodrenaje inmediato debió complementar la oclusión, evitando contracciones y riesgos en ese período. El acortamiento cervical $(7 \mathrm{~mm})$ y la protrusión de las membranas ovulares sugerían prematurez inminente, porque el aumento exponencial del riesgo en gemelos ocurre desde los $25 \mathrm{~mm}$ (40), esto hizo plantear la indicación de cerclage para prevenir el parto prematuro. Su indicación en incompetencia cervical es con menos de 25 semanas ya que tiene riesgo de amniorrexis y el cuello tenía consistencia intermedia, posición posterior, orificio externo cerrado, descartando el procedimiento por esas razones. La evolución posterior sin tocólisis, con edad de 36 semanas al parto demuestran que la decisión fue acertada.

3. Vía de parto. La cesárea tiene frecuencia alta por indicaciones propias del gemelar, pero no hay evidencias que apoyen un uso rutinario (41); tiene morbilidad significativamente mayor que en únicos $(42,43)$ y su prevención se inicia con la selección adecuada de los casos para el parto vaginal. La elección de la vía de parto es la última etapa del cuidado obstétrico y se ha racionalizado con el diagnóstico de corionicidad, precedente al criterio de presentación fetal lo que permite excluir de la opción vaginal a los casos inapropiados.

La cesárea es indiscutible en situaciones propias del embarazo gemelar en que el riesgo limita otra vía: gemelar con vasa previa, monocoriónicos monoamnióticos, monocoriónicos con STGG no tratados, monocoriónicos con restricción, dicoriónicos con restricción, siameses y múltiples de alto rango.

En gemelos con mejor placentación orgánica y funcional (bicoriónicos o monocoriónicos sin restricción ni transfusión) puede aplicarse el criterio de presentación-peso para indicar la vía; en los dos casos de monocoriónicos presentados se asumió con presentación cefálica del primer gemelo. Al nacimiento se dispuso de monitoreo continuo y concurrente de FCF por el mayor riesgo de asfixia $(31,44)$, vigilancia ultrasónica de presentación y posición del cordón (45); como también uso de ocitocina, para prevención de hipodinamia $(30 \%$ de los casos) (46) y anestesia epidural que tendría efecto beneficioso en el bienestar del segundo gemelo $(47,48)$. Es inadecuado considerar extemporáneamente la presentación a días del parto para determinar la vía porque puede variar al inicio de este, aún con examen reciente (49); en los casos presentados la presentación cefálica fue estable durante la dilatación y la tasa de cesárea en esta presentación es menor a $25 \%$ y sin complicación perinatal (50). El peso estimado al inicio del parto fue mayor a $2000 \mathrm{~g}$, sin restricción actual (es importante confirmarla por expresión tardía o ultrasonido distante), porque la discordancia se asocia a mayor mortalidad y es indicación de cesárea $(51,52,53,54)$. El ultrasonido en caso 1 
además se usó en vigilancia de Gem B (control de frecuencia cardiaca fetal y cordón vulnerable); en el caso 2 para observar la relación del Gem A con acardio que permaneció adosado a placenta y no constituyó factor obstructivo.

Ambas pacientes, primíparas, la conducción racional permitió parto vaginal, lo que es evidente en el partograma en las curvas de dilatación y progresión del descenso. La distocia de posición (OIDP) se resuelve por rotación espontánea, manual o fórceps (55). Las razones de elección fueron: evitar moldeamiento cefálico en feto de pretérmino por estación prolongada, prevenir detención en transversa por rotación insuficiente $\left(45^{\circ}\right)$, evitar dos maniobras si fracasa rotación manual y conducir expulsivo de menor duración porque Av-v abierta posteriormente al procedimiento de coagulación puede transfundir volumen intraparto. La rotación de $90^{\circ}$ a OP con conducción simple de cabeza hacia concavidad sacra y su desprendimiento fue inocua y evitó la tracción con el uso de ambas ramas. Las variables del parto, el alumbramiento, la condición neonatal y la evolución materna estuvieron dentro de las expectativas de la planificación y no fueron diferentes a las esperadas en parto único.

\section{CONCLUSIONES}

Los casos de la serie presentada demuestran:

1. La terapia in útero no complicada mejora la funcionalidad placentaria.

2. Esta mejoría es suficiente para el mayor requerimiento de intercambio feto-materno del trabajo de parto.

3. Hay tolerancia fetal a maniobras resolutivas de distocias en período expulsivo.

4. El nacimiento vaginal en dos primíparas mejora su pronóstico obstétrico por disponer de útero sin cicatriz.

5. El parto vaginal puede ser indicador de terapia exitosa.

6. El beneficio de las terapias actuales y con protocolo específico en Unidades Obstétricas de nivel terciario, el nacimiento vía vaginal puede ser recomendable para casos de gemelares monocoriónicos seleccionados.

AGRADECIMIENTOS: los autores dejan su constancia al Profesor Kipros Nikolaides por su inestimable aporte en el manejo del caso 1; a los Profesores A. Dabancens y A. Espinoza, por el estudio placentario y a la Profesora G. Henríquez por el estudio ecocardiográfico.

\section{BIBLIOGRAFÍA}

1. Hasbun J. Epidemiología del embarazo múltiple. REV Chil Obstet Ginecol 1998; 63(2): 129-31.

2. Hasbun J, Burgos N, Veloso D, Carrasco O. Complicaciones maternas del embarazo gemelar. XXIX Congreso Chileno de Obstetricia y Ginecología Libro de Resúmenes, 2001; 84-85.

3. Hasbun J. Manejo del parto gemelar. Urgencias y Complicaciones en Obstetricia. García-Huidobro M, Hasbun J, (Eds). Editorial Universitaria, 2004 (en prensa).

4. Bernirschke K. The contribution of placental anastomoses to prenatal twin damage. Hum Pathol 1992; 23: 1319-20.

5. Bajoria R, Wigglesworth J, Fisk NM. Angioarchitecture of monochorionic placentas in relation to the twin-twin transfusion syndrome. Am J Obstet Gynecol 1995; 172: 855-63.

6. Umur A, van Gemert MJ, Nikkels PGJ, Ross MG. Monochorionic Twins and twin-twin transfusion syndrome: the protective role of arterio-arterial anastomosis. Placenta 2002; 23: 201-09.

7. Sebire NJ, Snijders R, Hughes K, Sepulveda W, Nicolaides $\mathrm{KH}$. The hidden mortality of monochorionic twin pregnancies. Br J Obstet Gynecol 1997; 104: 1203-1207.

8. Machin G, Still K, Lalany T. Correlation of placental vascular anatomy and clinical outcomes in 69 monochorionic twin pregnancies. Am J Med Genet 1996; 61: 229-36.

9. Gratacos E, Van Schoubroeck D, Carreras E, Devlieger R, Roma E, Cabero L et al. Impact of laser coagulation in severe twin-twin transfusion syndrome on fetal Doppler indices and venous blood flow volume. Ultrasound Obstet Gynecol 2002; 20: 12530.

10. Ville $Y$, Hecher K, Gagnon A, Sebire N, Hyett J, Nicolaides K. Endoscopic laser coagulation in the management of severe twin transfusion syndrome Br J Obstet Gynecol 1998; 105 : 446-53.

11. De Lia JE, Kuhlman RS, Lopez KP. Treating previable twin-twin transfusion syndrome with fetoscopic laser surgery: outcomes following the learning curve. J Perinat Med 1999; 27: 61-7.

12. Quintero R, Comas C, Bornick M, Allen M, Kruger $M$. Selective versus non-selective laser photocoagulation of placental vessels in twin to twin transfusion syndrome. Ultrasound Obstet Gynecol 2000; 16(3): 230-36.

13. Quintero RA, Muñoz H, Hasbun J, Pommer R, Gutiérrez J, Sánchez J y col. Cirugía endoscópica fetal en un caso de embarazo gemelar complicado con secuencia de perfusión arterial reversa (secuencia TRAP). Rev Chil Obstet Ginecol 1995; 60(2): 112-17.

14. Sepulveda W, Sfeir D, Reyes M, Martinez J. Severe polydramnios in twin reversed arterial perfusion in sequence: successful management with intrafetal 
alcohol ablation of acardiac twin and amniodrenage. Ultrasound Obstet Gynecol 2000; 16: 260-63.

15. Quintero RA, Muñoz H, Pommer R, Díaz C, Bornicke PW, Allen MH. Operative fetoscopy via telesurgery. Ultrasound Obstet Gynec 2002; 20(4): 390-91.

16. Poblete AJ, Pomés C, Figueroa J, Soto J, Gana A, Montaña $\mathrm{R}$ y col. Cirugía láser endoscópica de la placenta en el tratamiento del síndrome de transfusión feto-fetal. Rev Chil Obstet Ginecol 2003; 68(6): 503-07.

17. Sepúlveda W, Corral E, Dezerega V, Devoto G, Schnapp C, Alcalde J. transfusión feto-fetal: Resultado perinatal exitoso con cirugía endoscópica láser. Rev Chil Ultrasonogr 2003; 4: 126-29.

19. Healey MG. Acardia: predictive risk factors for the co-twin's survival. Teratology 1994; 50: 205-213.

20. Sullivan A, Varner M, Ball R, Jackson M, Silver R. The management of acardiac twins: A conservative approach. Am J Obstet Gynecol 2003; 189: 1310-13.

21. Moore TR, Gale S, Benirscke K. Perinatal outcome of forty-nine pregnancies complicated by acardiac twinning. Am J Obstet Gynecol 1990; 163: 907-12.

22. Dashe JS, Fernandez CO, Twicler DM. Utility of Doppler velocimetry in predicting outcome in twin reversed arterial perfusion sequence. Am J Obtet Gynecol 2001; 185: 135-39.

23. Sepulveda W, Bower S, Hassan J, Fisk N. Ablation of acardiac twin by alcohol injection into the intraabdominal umblical artery. Obstet Gynecol 1995; 86: 680-81

24. TY Tan, Sepulveda W. Acardiac twin: a systematic review of minimally invasive treatment modalities. Ultrasound Obstet Gynecol 2003; 22: 409-19.

25. Sepúlveda W, Hasbun J, Dezerega V, Devoto J, Alcalde J. Successful sonographical guided laser ablation of a large acardiac twin at 26 weeks gestation. J Ultrasound Med 2004; 23: 1663-66.

26. Deprest J, Lerut T, Vanderberghe K. Operative fetoscopy: new perspective in fetal therapy? Prenatal Diagnosis 1997; 17(13) : 1247-60.

27. Williams K, Hennessy E, Albernan B. Cerebral palsy: effects of twinning, birthweight, and gestational age. Arch Dis Child 1996; 75: F178-82.

28. Sutcliffe AG, Sebire NJ, Pigott AJ, Taylor B, Edwards $\mathrm{PR}$, Nicolaides $\mathrm{KH}$. Outcome for children born after in uterus laser ablation therapy for severe twin-totwin transfusion syndrome. BJOG 2001; 108: 124650.

29. Hasbun J, Valenzuela P, Veloso D, Burgos N. Prematurez en embarazo gemelar. XXIX Congreso Chileno de Obstetricia y Ginecología Libro de Resúmenes, 2001; 86-7.

30. Gardner MO, Goldenberg R, Cliver S, Tucker J, Nelson K, Cooper R: The origin and the outcome of preterm twin or singleton. Obstet Gynecol 1994; 85: 553-56.

31. Kiely JL. The epidemiology of perinatal mortaly in multiple births. Bull NY Acad Med 1990; 66: 618-37.
32. Newman RB, Campio S, Gill PJ, Katz M. The influence of fetal number on antepartum uterine activity. Obstetric Gynecol 1989; 73: 696-99.

33. Newman RB, Gill PJ, Katz M. Uterine activity during pregnancy in ambulatory patients: comparison of singleton and twin gestations. Am J Obstet Gynecol 1986; 154: 530-1.

34. Garite TJ, Bentley D, Hamer CH, Porto M. Uterine activity characteristics in multiple gestations. Obstet Gynecol 1990; 76: 56S.

35. Dyson AC, Cristes YM, Ray DA. Prevention of preterm birth in high risk patients: the role of education and provider contact versus home monitoring. Am J Obstetric Gynecol 1991; 164: 756-62.

36. Bell $R$. The prediction of preterm labor by recording spontaneous antenatal uterine activity. $\mathrm{Br} \mathrm{J}$ Obstet Gynecol 1983; 90: 884-89.

37. Morrison JC, Martín Jr JN, Martín RW, Gookin KS, Wiser WL. Prevention of preterm birth by ambulatory assessment of uterine activity: a randomized study. Am J Obstet Gynecol 1987; 156: 536-43.

38. lams JD, Johnson FF, O'Shaughnessy RW. A prospective random trial of home activity monitoring in pregnancies at increased risk of preterm labor. Am J Obstet Gynecol 1988; 159: 595-603.

39. Moore TR, lams JD, Cresy MD, Burau KD. The uterine activity in pregnancy Workshop Group. Obstetric Gynecol 1994; 83(4): 517-23.

40. Skentou C, Souka AP, To MS, Liao AW, Nicolaides $\mathrm{KH}$. Prediction of preterm delivery in twins by cervical assessment at 23 weeks. Ultrasound Obstet Gynecol 2001; 17: 7-10.

41. Hogle K, Hutton E, Mc Brien K, Barret J, Hannah M. Cesarean delivery for twins: A systematic review and meta-analysis. Am J Obstetric Gynecol 2003; 188: 220-27.

42. Herruzo AJ. Perinatal morbidity and mortality in twin pregnancies labor. Acta Genet Med Gemellol (Roma) 1979; 28: 303-09.

43. Suonio S, Huttunen M. Puerperal endometritis after abdominal twin delivery. Acta Obstet Gynecol Scand 1994; 7: 26-7.

44. Goldenberg RL, Nelson KG, Burton M, Anderson $\mathrm{JH}$, Waine JB. Gestational age and the management preterm labor in obstetrical programs. Am J Perinatol 1985; 2: 25-9.

45. Gurewitsch E, Skupski W, Chevernak F. The role of ultrasonography in the intrapartum management of multiple gestations. In ultrasound and multifetal pregnancy. The Parthenon Publishing Group. London. 1998. 153-63.

46. Leroy F. Oxytocin treatment in twins pregnancy labor. Acta Genet Med Gemellol 1979; 28: 303-309.

47. Crawford JS. A prospective study of 200 consecutive twins delivery. Anaesthesia 1987; 42: 33-43.

48. Eskes T. The second twin. Eur J Obstet Gynecol Reprod Biol 1985; 19: 159-66.

49. Divon MY, Marin MJ, Pollack RN, Katz NT, Henderson C, Aboulafia $Y$ et al. Twins gestation: 
fetal presentation as a function of gestational age. Am J Obstet Gynecol 1993; 168: 1500-02.

50. Chauan S, Roberts W. Intrapartum Management. In: Gall SA (ed). Multiple Pregnancy and Delivery. St Louis: Mosby. USA,1996; 243-80.

51. Chevernak FA, Johnson RE, Youcha S, Hobbins J Berkowitz RL. Intrapartum management of twin gestation. Obstet Gynecol 1985; 65: 119-24.

52. Olofsson P, Rydhstrom H. Twin delivery: how should the second twin be delivered? Am J. Obstet Gynecol 1985; 153: 479-81.

53. Chauan S, Washburne JF, Martin JN, Robert WE,
Roah H, Morrison JC. Intrapartum assessment by house staff of birth weight among twins. Obstet Gynecol 1993; 82: 523-26.

54. Vergani P, Locatelli A, Ratti M, Scian A, Pozzi E, Pezzullo $\mathrm{J}$ y cols. Preterm twins: What threshold of birth weight discordance heralds majors adverse neonatal outcome? Am J Obstetrics Gynecol 2004; 191: 1441-45.

55. King E. Transverse arrest and occipitosacral positions. Charles Thomas (ed). Banner Stone House. Illinois. Blackwell Scientific Publications Ltd. Oxford, 1957, 91-99. 